\title{
Bandwidth-Efficient Pilot Symbol Aided Technique for Trellis-Coded Modulation in Land Mobile Satellite Fading Channels
}

\author{
$\because \quad$ M. H. Ng and S. W. Cheung \\ Department of Electrical and Electronic Engineering \\ The University of Hong Kong \\ Hong Kong \\ Phone: (852) 2859-2711 \\ Fax: (852) 2559-8738 \\ Email: mhng@eee.hku.hk and swcheung@eee.hku.hk
}

\begin{abstract}
A bandwidth-efficient pilot-symbol-aided technique is proposed for use with trellis-coded modulation (TCM). Computer simulation has been used to assess the effects of the technique on the bit error rate (BER) performances of trelliscoded 16-ary quadrature amplitude modulation (TC-16QAM) in the flat Rician and shadowed Rician fading channels corrupted with additive white Gaussian noise. The results have shown that the proposed technique needs a very low bandwidth redundancy and can work effectively at low signal-to-noise ratios, so it is suitable for use with TCM.
\end{abstract}

\section{INTRODUCTION}

Pilot symbol aided (PSA) transmission is a very effective method to combat multipath fading in the mobile radio environments [1-4]. In a PSA system, redundant bandwidth is required to transmit the pilot symbols. In a fast fading environment with $f_{D} T=5 \times 10^{-2}$, where $f_{D}$ is the maximum Doppler spread and $T$ is the channel symbol period, the bandwidth redundancy can be as high as $20 \%$ [1]. A novel technique that employs both of the data and pilot symbols for fading estimation has been proposed to significantly reduce the bandwidth redundancy of the PSA systems [4]. However, the teclunique only works effectively at high signal-to-noise ratios (SNRs), but is inferior at low SNRs. In systems where trellis-coded modulation (TCM) is used, the operating SNRs are normally in the lower end and so the PSA technique also has to work effectively at low SNRs. The teclinique, therefore, becomes less attractive for use with TCM.

A bandwidth-efficient PSA technique that can work effectively at low SNRs is proposed in this paper. Monte Carlo computer simulation has been performed to assess the effectiveness of the technique on the bit error rate (BER) performances of trellis-coded 16-ary quadrature amplitude modulation (TC-16QAM) in the flat Rician and shadowed Rician fading channels corrupted with additive white Gaussian noise (AWGN). The results have shown that, in a fast fading environment with $f_{D} T=5 \times 10^{-2}$, the technique requires a bandwidth redundancy of only $6.67 \%$, yet achieving good performances at low SNRs.

\section{Proposed psa technique}

The system model used in this study is shown in Fig. 1. In the transmitter, the data are first trellis encoded and then block interleaved. The TC-16QAM encoder has a rate of $3 / 4$ and eight states [5] and the interleaver has a depth of 10 rows and a span of 8 columns. A pilot symbol from a known pseudo-random sequence is multiplexed with every $(L-I)$ data symbols to form a pilot frame of $L$ symbols [1-4]. The transmitted signal is fed to a flat Rician or shadowed Rician fading channel corrupted with AWGN. The fading models are described in [6].

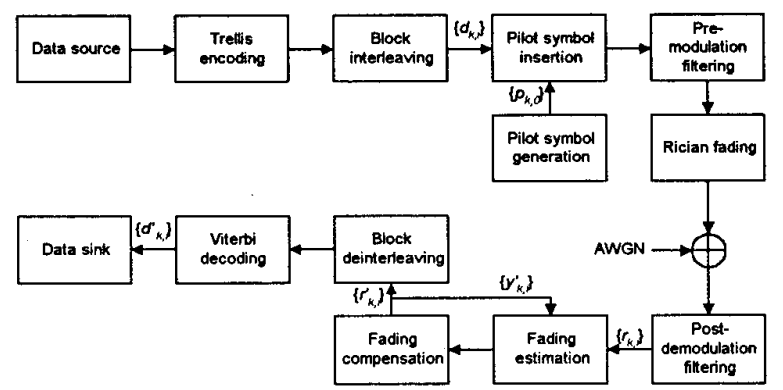

Fig. 1. System model.

At the receiver, after matched filtering, the received signal is sampled in synchronism at time $t=i T$ seconds to give the $i$-th symbol of the $k$-th frame as

$$
r_{k, i}=q_{k, i} y_{k, i}+w_{k, i}
$$

where $q_{k, i}$ is the transmitted pilot or data symbol, and $y_{k, i}$ and $w_{k, i}$ are the fading effect and noise sample, respectively.

The fading effects, $y_{k, 0}$ and $y_{k+1,0}$, on the pilot symbols, $p_{k, 0}$ and $p_{k+1,0}$, of the $k$-th and $(k+1)$-th frames, respectively, are estimated as 


$$
\begin{aligned}
& \hat{y}_{k, 0}=\frac{r_{k, 0}}{p_{k, 0}}=y_{k, 0}+\frac{w_{k, 0}}{p_{k, 0}} \\
& \text { and } \quad \hat{y}_{k+1,0}=\frac{r_{k+1,0}}{p_{k+1,0}}=y_{k+1,0}+\frac{w_{k+1,0}}{p_{k+1,0}}
\end{aligned}
$$

The fading effect $y_{k, i}$ is then estimated using linear interpolation on $\hat{y}_{k, 0}$ and $\hat{y}_{k+1,0}$ as [2]

$$
\hat{y}_{k, i}=\frac{(L-i)\left(y_{k, 0}+w_{k, 0} / p_{k, 0}\right)+i\left(y_{k+1,0}+w_{k+1,0} / p_{k+1,0}\right)}{L}
$$

Alternatively, $y_{k, i}$ can be estimated by minimizing the square of the symbol estimation errors as [4]

$$
\begin{aligned}
\tilde{y}_{k, i} & =\frac{(i-m) \tilde{y}_{k, i+1}+\widetilde{y}_{k, m}}{i-m+1} \\
\text { or } \quad \tilde{y}_{k, i} & =\frac{(n-i) \tilde{y}_{k, i-1}+\widetilde{y}_{k, n}}{n-i+1}
\end{aligned}
$$

where $\widetilde{y}_{k, m}$ and $\widetilde{y}_{k, n}$ are the fading estimates on the $m$-th and $n$-th symbols, respectively, and $m<i<n$.

The two fading estimates, $\hat{y}_{k, i}$ and $\dot{\tilde{y}}_{k, i}$, are used to compensate for the fading effect on $r_{k, i}$ to give the respective symbols

$$
\begin{aligned}
\hat{r}_{k, i} & =\frac{r_{k, i}}{\hat{y}_{k, i}} \\
\text { and } \quad \tilde{r}_{k, i} & =\frac{r_{k, i}}{\widetilde{y}_{k, i}}
\end{aligned}
$$

The symbols $\hat{r}_{k, i}$ and $\tilde{r}_{k, i}$ are weighted and added as

$$
r_{k, i}^{\prime}=\frac{\hat{r}_{k, i} \hat{z}_{k, i}+\widetilde{r}_{k, i} \widetilde{z}_{k, i}}{\hat{z}_{k, i}+\widetilde{z}_{k, i}}
$$

where $\hat{z}_{k, i}$ and $\widetilde{z}_{k, i}$ are the weighting factors obtained using the estimated SNRs of $\hat{r}_{k, i}$ and $\widetilde{r}_{k, i}$, respectively, as

$$
\hat{z}_{k, i}=\frac{\left|\hat{y}_{k, i}\right|^{2}}{\left|r_{k, i}-\hat{y}_{k, i} \hat{d}_{k, i}\right|^{2}}
$$$$
\text { and } \quad \widetilde{z}_{k, i}=\frac{\left|\widetilde{y}_{k, i}\right|^{2}}{\left|r_{k, i}-\widetilde{y}_{k, i} \tilde{d}_{k, i}\right|^{2}}
$$

where $\hat{d}_{k, i}$ and $\widetilde{d}_{k, i}$ are the vectors closest to $\hat{r}_{k, i}$ and $\widetilde{r}_{k, i}$, respectively, in the 16QAM signal constellation. Equation (9) clearly shows that the combining process accentuates the signal with a higher SNR and suppresses the one with a lower SNR.

\section{$\widetilde{y}_{k, i}$ is then re-estimated as}

$$
y_{k, i}^{\prime}=\frac{\hat{y}_{k, i} \hat{z}_{k, i}+\left(r_{k, i} / \widetilde{d}_{k, i}\right) \widetilde{z}_{k, i}}{\hat{z}_{k, i}+\widetilde{z}_{k, i}}
$$

which is used as $\widetilde{y}_{k, i+1}, \widetilde{y}_{k, m}, \widetilde{y}_{k, i-1}$ or $\widetilde{y}_{k, n}$ in (5) and (6) to estimate the fading effect on the next data symbol of the same frame [4].

The combined symbol $r_{k, i}^{\prime}$ is then deinterleaved and fed into the Viterbi decoder to give the output data symbol $d_{k, i}^{\prime}$. The decision depth of the Viterbi decoder is 32 symbols, and the maximum likelihood decoding metric is given by [3]

$$
m\left(r_{k, i}^{\prime}, d_{k, i}^{\prime} ; u_{k, i}\right)=-u_{k, i}\left|r_{k, i}^{\prime}-d_{k, i}^{\prime}\right|^{2}
$$

where the weighting factor $u_{k, i}$ is obtained as

$$
u_{k, i}=\frac{\left|\hat{y}_{k, i}\right|^{2} \hat{z}_{k, i}+\left|\widetilde{y}_{k, i}\right|^{2} \widetilde{z}_{k, i}}{\hat{z}_{k, i}+\widetilde{z}_{k, i}}
$$

This whole process continues until all data symbols within the received signal have been detected.

\section{RESUlts AND DISCUSSIONS}

Monte Carlo computer simulation has been used to study the BER performances of the proposed technique on TC16QAM. The normalized Doppler spreads of $f_{D} T=5 \times 10^{-2}$ and $2.5 \times 10^{-2}$ have been used in the tests. For the purpose of comparison, the results using linear interpolation on the pilot symbols for fadin estimation and compensation are also present. These resuits are indexed by a '[P]' in the legend of the Figures.

With a Doppler spread of $f_{D} T=5 \times 10^{-2}$, the BER performances of TC-16QAM in a Rician fading channel with signal-to-multipath ratio (SMR) $=7 \mathrm{~dB}$ and frame lengths $L=$ 5, 10 and 15 are shown in Fig. 2. To illustrate the shortcoming of the technique proposed in [4], the results using the technique are also shown and indexed by a '[D]' in the same figure. It can be seen that, when compared with the results using linear interpolation, the technique is only 
superior at higher SNRs but inferior at lower SNRs. Thus at lower SNRs, the technique is not suitable for the system. However, Fig. 2 shows that the technique proposed in this paper can achieve either better or equal performances under all conditions tested. The performances are considerably better at higher SNRs or with longer frame lengths. With $L=$ 15 , the technique starts gaining advantages at a SNR of about $5 \mathrm{~dB}$ and can greatly reduce the BER from $3 \times 10^{-2}$ to $6 \times 10^{-3}$ at $\mathrm{SNR}=30 \mathrm{~dB}$. Here the required bandwidth redundancy is only $6.67 \%$.

The BER performances of TC-16QAM in light and average shadowed Rician fading channels, with $f_{D} T=5 \times 10^{-2}$ and $L$ $=5,10$ or 15, are shown in Figs. 3 and 4, respectively. The BER performances of TC-16QAM in a heavy shadowed Rician fading channel with a slower fading rate of $f_{D} T=2.5 \times 10^{-2}$ and $L=10,15$ or 20 are shown in Fig. 5 . Here again, with shorter frame lengths, the proposed technique has about the same performances as linear interpolation at lower SNRs. With longer frame lengths, the technique achieves notably better performances than those of linear interpolation at higher SNRs. Therefore, in the bandwidth-limited TCM systems where low bandwidth redundancies, i.e. long frame lengths, are required, the proposed PSA technique is obviously better.

\section{Conclusions}

A bandwidth-efficient fading estimation technique for use with TCM has been proposed and studied. The technique employs both data and pilot symbols for fading estimation. Computer simulation results have shown that in the flat Rician and shadowed Rician fading channels, the proposed technique outperforms linear interpolation at low SNRs, yet requiring low bandwidth redundancies, so it is suitable for use with TCM.
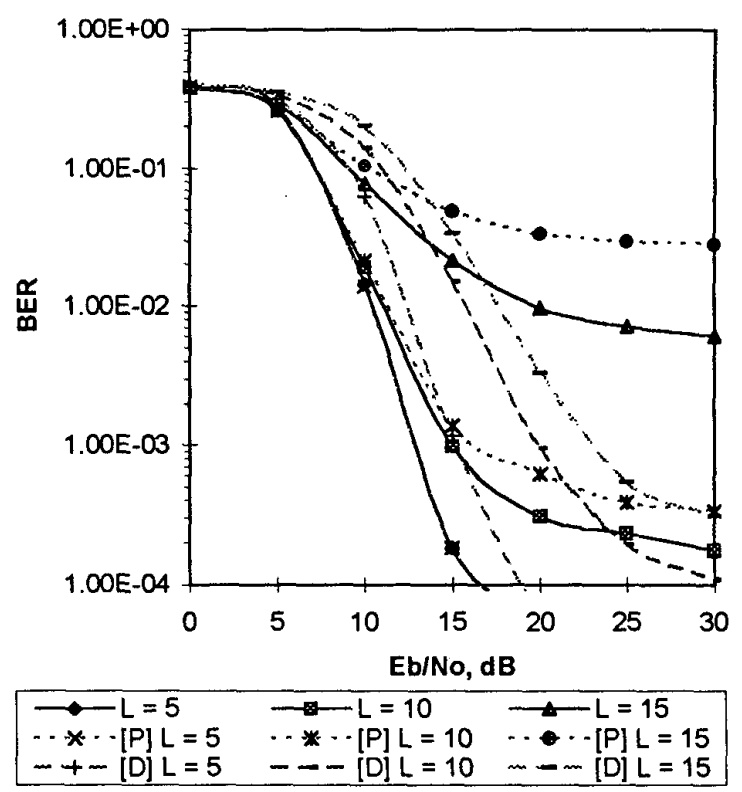

Fig. 2. BER performances in a Rician fading channel with $\mathrm{SMR}=7 \mathrm{~dB}, f_{D} T=5 \times 10^{-2}$ and $L=5,10$ or 15.
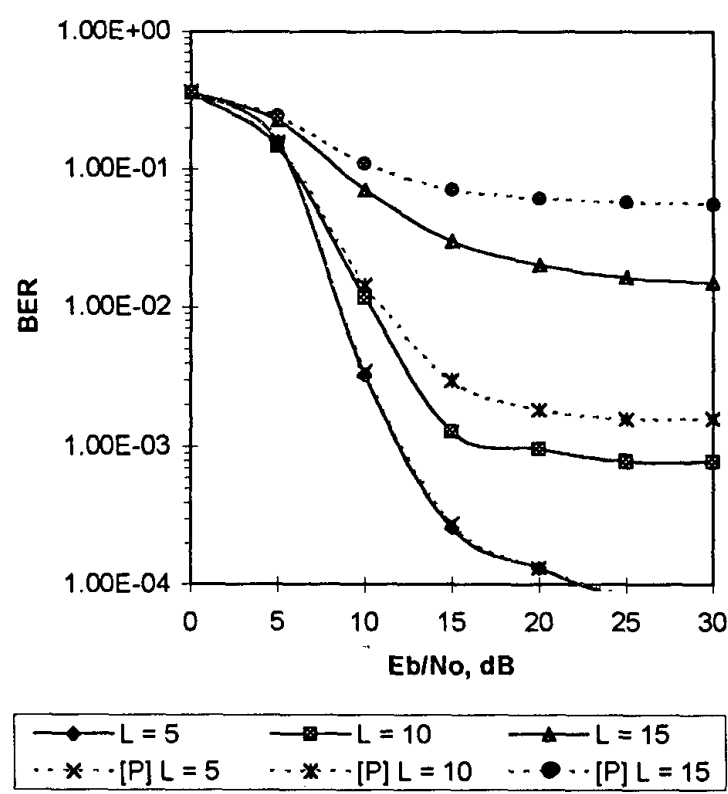

Fig. 3. BER performances in a light shadowed Rician fading channel with $f_{D} T=5 \times 10^{-2}$ and $L=5,10$ or 15 . 


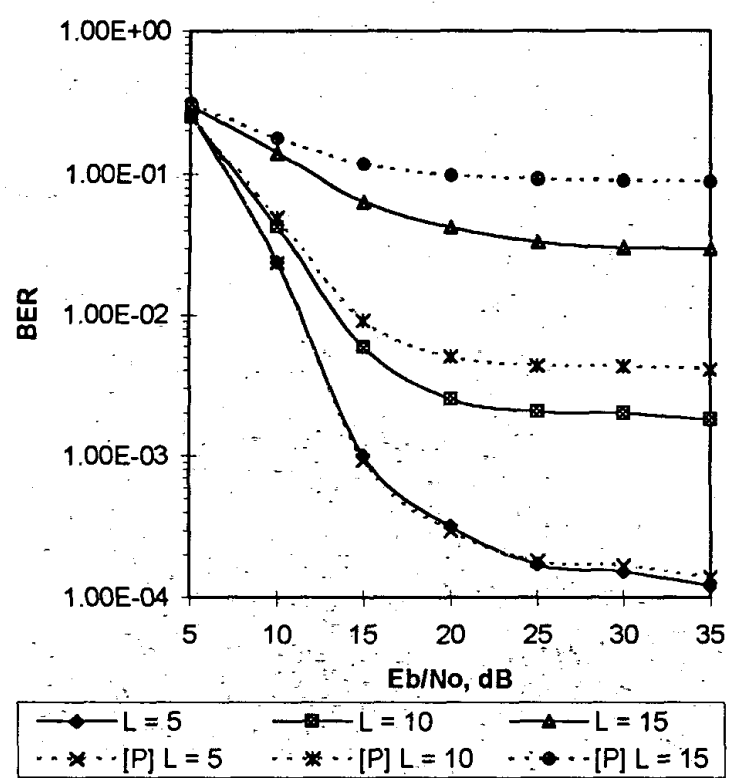

Fig. 4. BER performances in an average shadowed Rician fading channel with $f_{D} T=5 \times 10^{-2}$ and $L=5,10$ or 15 .

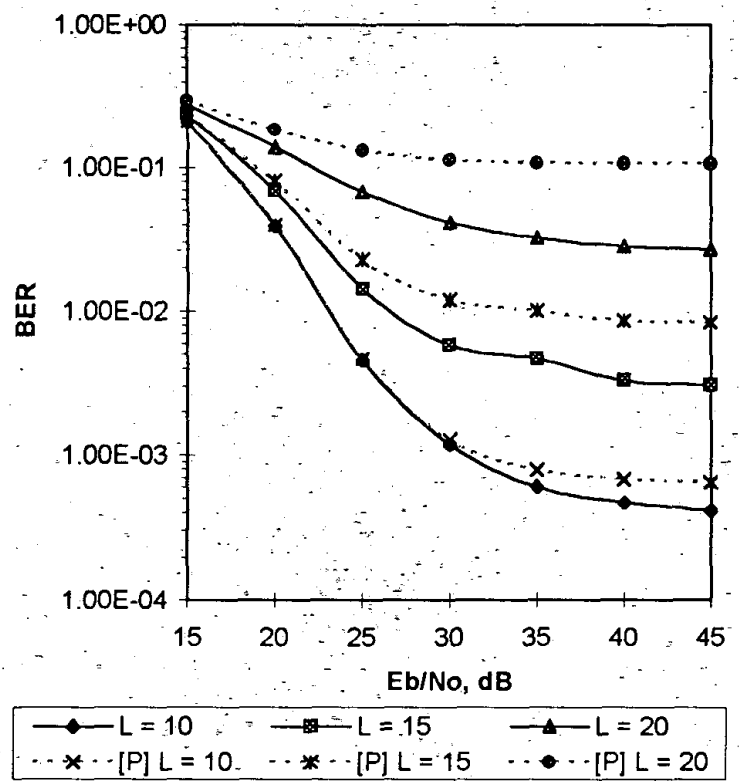

Fig. 5. BER performances in a heavy shadowed Rician fading channel with $f_{D} T=2.5 \times 10^{-2}$ and $L=10,15$ or 20 .

\section{REFERENCES}

[1] G. T. Irvine and P. J. McLane, "Symbol-aided plus decision-directed reception for PSK/TCM modulation on shadowed mobile satellite fading channels," IEEE $J$. Select. Areas Commun., vol. 10, no. 8, pp. 1289-1299, Oct. 1992.

[2] S. Sampei and T. Sunaga, "Rayleigh fäding compensation for QAM in land mobile radio communications," IEEE Trans. Veh. Technol., vol. 42, no. 2, pp. 137-147, May 1993.

[3] K. Y: Tsie and A. H. Aghvami, "High level trellis-coded modulation with slow frequency hopping for land mobile communications," IEEE Trans. Veh. Technol.; vol. 43, no. 1, pp. 147-155, Feb. 1994.

[4] . M. H. Ng and S. W. Cheung, "Bandwidth-efficient pilot symbol aided technique," Electronics Letters, vol. 34, no. 16, pp. 1548-1550, Aug. 1998.

[5] E. Bilglieri, D. Divsalar, P. J. McLane, and M. K. Simon, Introduction to - Trellis-Coded Modulation with Applications. New York: Macmillan, 1991, pp. 164-167.

[6] C. Loo and N. Secord, "Computer models for fading channels with applications to digital transmission," IEEE Trans. Veh. Technol., vol. 40, pp. 700-707, Nov. 1991. 